\title{
A new implant device to prevent edema-associated underdosage in high-dose-rate interstitial brachytherapy of mobile tongue cancer
}

\author{
Ken Yoshida, MD, PhD', Tadayuki Kotsuma, MD², Hironori Akiyama, DDS³, Hideya Yamazaki, MD4,

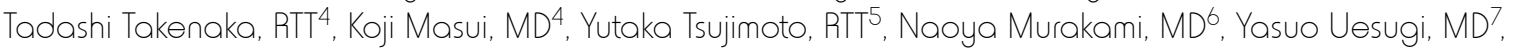 \\ Taiju Shimbo, MD!, Nobuhiko Yoshikawa, MD!, Hiroto Yoshioka, MD!, Mio Nakata, MD!, Takumi Arika, DDS, \\ Yuji Takaoka, MD², Eiichi Tanaka, MD², Nikolaos Tselis, MD? \\ 'Department of Radiation Oncology, Osaka Medical College, Takatsuki, Osaka, Japan, ${ }^{2}$ Department of Radiation Oncology, National \\ Hospital Organization Osaka National Hospital, Osaka, Japan, ${ }^{3}$ Department of Oral Radiology, Osaka Dental University, Osaka, \\ Japan, ${ }^{4}$ Department of Radiology, Kyoto Prefectural University of Medicine, Kyoto, Japan, ${ }^{5}$ Department of Radiology, National Hospital \\ Organization Osaka National Hospital, Osaka, Japan, 'Department of Radiation Oncology, National Cancer Center Hospital, Japan, \\ ${ }^{7}$ Department of Rehabilitation Sciences, Faculty of Allied Health Sciences, Kansai University of Welfare Sciences, Japan, ${ }^{8}$ Department of Oral \\ Surgery, National Hospital Organization Osaka National Hospital, Osaka, Japan, ${ }^{9}$ Department of Radiation Oncology, Johann Wolfgang \\ Goethe University Frankfurt, Germany
}

\begin{abstract}
Purpose: Tongue edema is a potential cause of treatment target underdosage in high-dose-rate interstitial brachytherapy (HDR-ISBT) of mobile tongue cancer. To prevent such edema-associated alteration of dosimetry, we developed a special silicon device. In this report we communicate our initial experience with two mobile tongue cancer patients whom we treated using this new device.

Material and methods: The device consists of silicone tubes with a fixed width and scalable length depending on tongue size. These tubes are lined and fixed like a palisade, allowing the device to be used also as a template. The device is placed next to the lateral border of the tongue and on the floor of the mouth. In addition, a vinyl template can be placed on the dorsal tongue surface with both devices combined for implantation guidance. Between June and August 2012, two patients with locally confined tongue cancer were treated.

Results: Between June and August 2012, two mobile tongue cancer patients classified as cT2N0M0 were treated with HDR-ISBT using the silicone device. They underwent ISBT as monotherapy with fractional doses of 6.0 Gy up to a total physical dose of $54.0 \mathrm{~Gy}$. The $\mathrm{D}_{90}(\mathrm{CTV})$ values of both patients were $6.3 \mathrm{~Gy}$ and $6.6 \mathrm{~Gy}$ and the $\mathrm{D}_{2 \mathrm{cc}}$ (mandible) values were $3.4 \mathrm{~Gy}$ and 2.6 Gy, respectively. At present, both patients remain without local disease recurrence at 60 and 56 months after ISBT, respectively.

Conclusions: The described silicone device has the potential to prevent underdosage to the treatment target related to tongue edema. It has been shown to be safe and easy to implement.

Key words: tongue edema, silicone device, high-dose-rate interstitial brachytherapy, mobile tongue cancer.

\section{Purpose}

High-dose-rate interstitial brachytherapy (HDR-ISBT) $[1,2,3,4]$ is an established radiotherapy (RT) modality for the treatment of tongue cancer. Computed tomography (CT)-based dosimetry will clarify in HDR-ISBT if there is any alteration of the geometrical relationship between interstitial catheter positions and the clinical target volume (CTV), which can occur during fractionated treatments. In analogy to this scenario, edema and applicator dis-

placement are potential causes of under- or overdosage of the CTV, as well as the organs at risk (OAR), in prostate and gynecologic HDR-ISBT $[5,6,7,8,9]$. Similarly, tongue edema can impair target coverage in temporary interstitial tongue RT $[10,11,12,13]$. To prevent such alteration of CTV coverage, we introduced a new device which aims to avoid an edema-associated shift of tongue tissue (mucosal surface) beyond the prescription isodose line. In this report we communicate our initial clinical experience with two mobile tongue cancer patients whom we treated
\end{abstract}


with HDR-ISBT using the new silicone device in order to prevent treatment-related tongue edema.

\section{Material and methods}

\section{Silicone device}

Our treatment device consists of silicone tubes with a fixed width $(5 \mathrm{~mm}$ outer diameter and $3 \mathrm{~mm}$ internal diameter) and a scalable length of 10-15 mm depending on tongue size. These tubes are lined and fixed at both ends with two pieces of string in a transverse direction like a palisade, which allows the device to be used as a template (Figure 1A). In the oral cavity the device is positioned next to the lateral border of the tongue and on the floor of the mouth. This also enables the lateral border of the tongue to be irradiated adequately by implanting catheters into every three silicone tubes, ensuring an applicator-applicator interval of about $15 \mathrm{~mm}$. In addition, a vinyl template can be placed on the dorsal tongue surface (Figure 1B) with both devices combined for the purpose of implantation guidance (Figure 1C).

\section{Implantation}

Clinical implementation of the new silicon device is part of our "classical" tongue implantation procedure. In short, open-end catheters (Bevel needle; Elekta AB, Stockholm, Sweden) are implanted from the submandibular region into the oral cavity. To treat adequately the lateral side of the tongue, the catheters are implanted into the floor of the mouth close to the lateral border of the
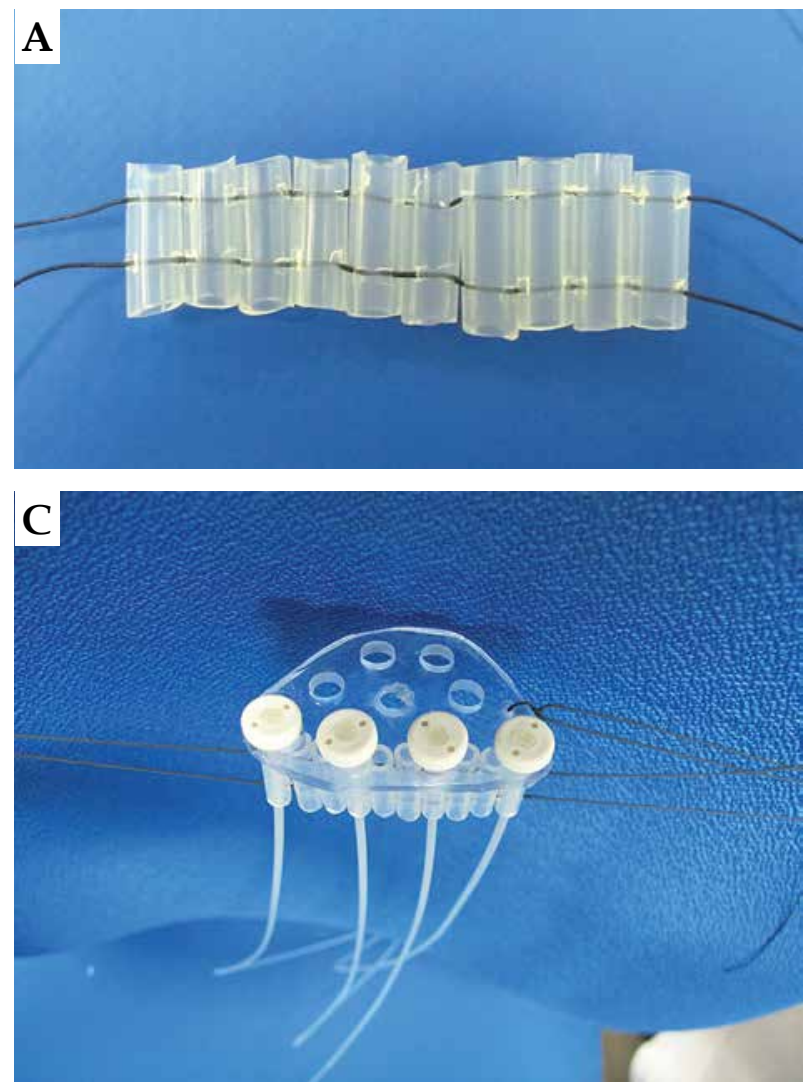

tongue (Figure 2A). The silicone device is at first placed on the floor of the mouth and the open-end catheters are penetrated from the floor surface into the silicone device and finally into the holes of the vinyl template. After completion of implantation, all open-end catheters are replaced by flexible applicator tubes (Flexible implant tube, 6 F/1.9 mm external diameter, single leader; Elekta AB) and these tubes fix silicon devices in a longitudinal direction. For medial parts of the tongue, open-end catheters are implanted through the dorsal surface of the tongue into the vinyl template and replaced by flexible applicator tubes (Figure 2B). Concerning the movement of flexible applicator tubes inside the silicon tubes, the applicators are fixed longitudinally because flexible applicator tubes penetrate tissue from the submandibular region to the mouth floor and the tubes are fixed by the button and vinyl template at the dorsum of the tongue. Laterally the maximum distance of this movement is $0.55 \mathrm{~mm}$ because the external diameter of the flexible applicator tubes is $1.9 \mathrm{~mm}$ and the internal diameter of the silicon tubes is $3 \mathrm{~mm} .0 .55 \mathrm{~mm}$ is a very small number and we consider that it is not necessary to take account into the movement of flexible applicator tubes inside silicon tubes for treatment planning. Before implantation, titanium markers are implanted at the edge of the gross tumor to aid CTV delineation for image-based treatment planning.

\section{Planning and treatment}

Our three-dimensional (3D) treatment planning method has been described elsewhere $[14,15]$. In short,



Fig. 1. A) Photograph of a silicone device. The width of the silicone tube was $5 \mathrm{~mm}$ outer diameter and $3 \mathrm{~mm}$ internal diameter, and its length was 10-15 mm depending on tongue size. These tubes were lined and fixed at both ends with two pieces of string in a transverse direction like a palisade. This device was positioned next to the lateral border surface of the tongue and on the floor of the mouth. We could use the device as a template. B) Photograph of a vinyl template. It was placed on the dorsal tongue surface. C) Photograph of combination with a silicone device and vinyl template. Flexible treatment applicator tubes (Flexible implant tube, 6 F/1.9 mm external diameter, single leader; Elekta AB, Stockholm, Sweden) were inserted 

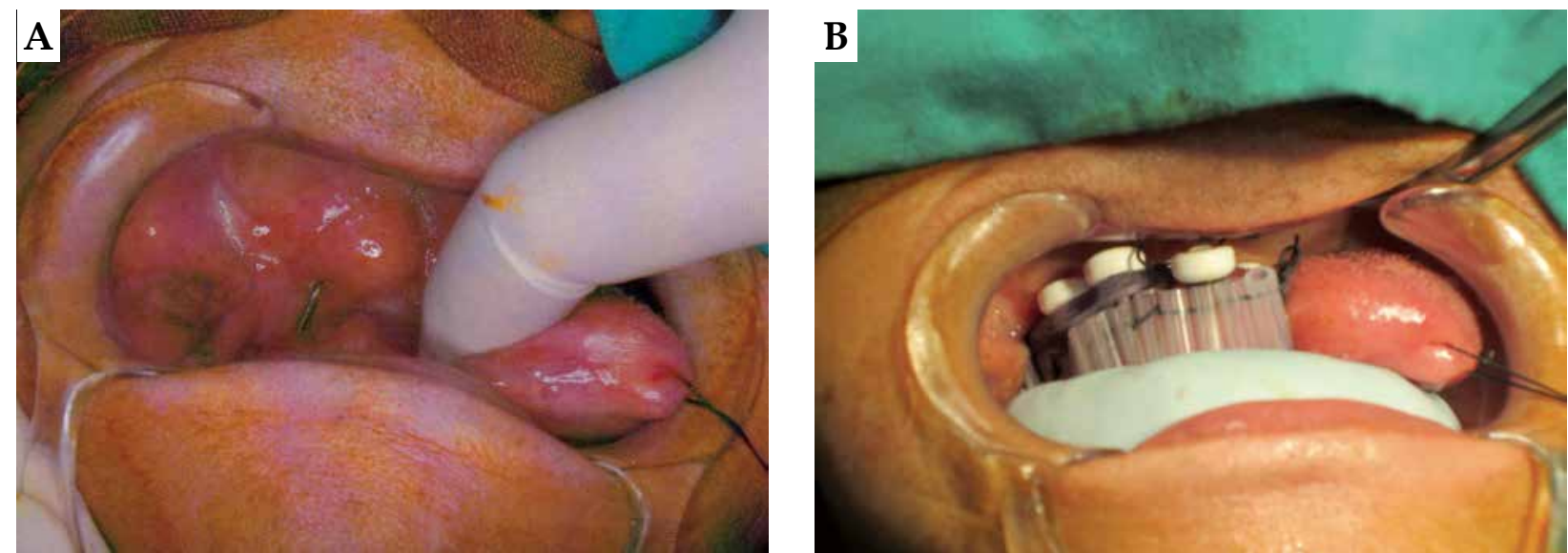

Fig. 2. A) Photograph of applicator implantation for case 1. Metallic open-end needle (Bevel needle; Elekta AB, Stockholm, Sweden) was implanted from submandibular region to oral cavity. B) Photograph of the same patient as Fig. 2A. Flexible treatment applicator tubes (Flexible implant tube, 6 F/1.9 mm external diameter, single leader; Elekta AB, Stockholm, Sweden) were implanted into the silicone device and vinyl template. We also inserted a silicone block between the lateral border of the tongue and gingiva

treatment planning is CT-based. We draw gross tumor volume (GTV), CTV and mandible as an OAR. CTV results from GTV plus at least a $5.0 \mathrm{~mm}$ margin and incorporates clinical findings from inspection, palpation, and intraoperative intra-oral ultrasound as well as titanium marker orientation. Planning is based on a modified Paris system approach with anatomy-oriented dose optimization (Figure 3A) [16]. Treatment plan evaluation is based on dose-volume histogram (DVH) analysis under consideration of the dose that covers $90 \%\left[\mathrm{D}_{90}(\mathrm{CTV})\right]$ as well as $100 \%$ of the CTV $\left[\mathrm{D}_{100}(\mathrm{CTV})\right]$. Our dosimetric goal is $\mathrm{D}_{100}$ $(\mathrm{CTV}) \geq$ prescription dose (PD); however, excessive doses to OARs (e.g. mandible) or a hyperdose sleeve $>10 \mathrm{~mm}$ allow for $\mathrm{D}_{100}(\mathrm{CTV})<\mathrm{PD}$. In such cases, our dosimetric goal is $\mathrm{D}_{90}(\mathrm{CTV})>\mathrm{PD}$. For DVH-based OARs evaluation, we use the minimum dose received by the maximally irradiated $2 \mathrm{cc}\left(\mathrm{D}_{2 \mathrm{cc}}\right)$ of the mandible. For this, we use lead blocks as spacers and shielding devices to the gingival mucosa and mandible for protection purposes [17]. For CT planning, however, we use silicone blocks instead of lead blocks to prevent metal artifacts (Figure 2B).

Our ISBT protocol consists in the monotherapy setting of fractional HDR doses of 6.0 Gy up to a total PD of $54.0 \mathrm{~Gy}$ in nine fractions over 7 days. Two fractions were administered per day. The time interval between fractions was $>6 \mathrm{~h}$. The implantation and first treatment were done on Wednesday, and the final treatment and the extraction of the flexible applicator tubes on the next Tues-
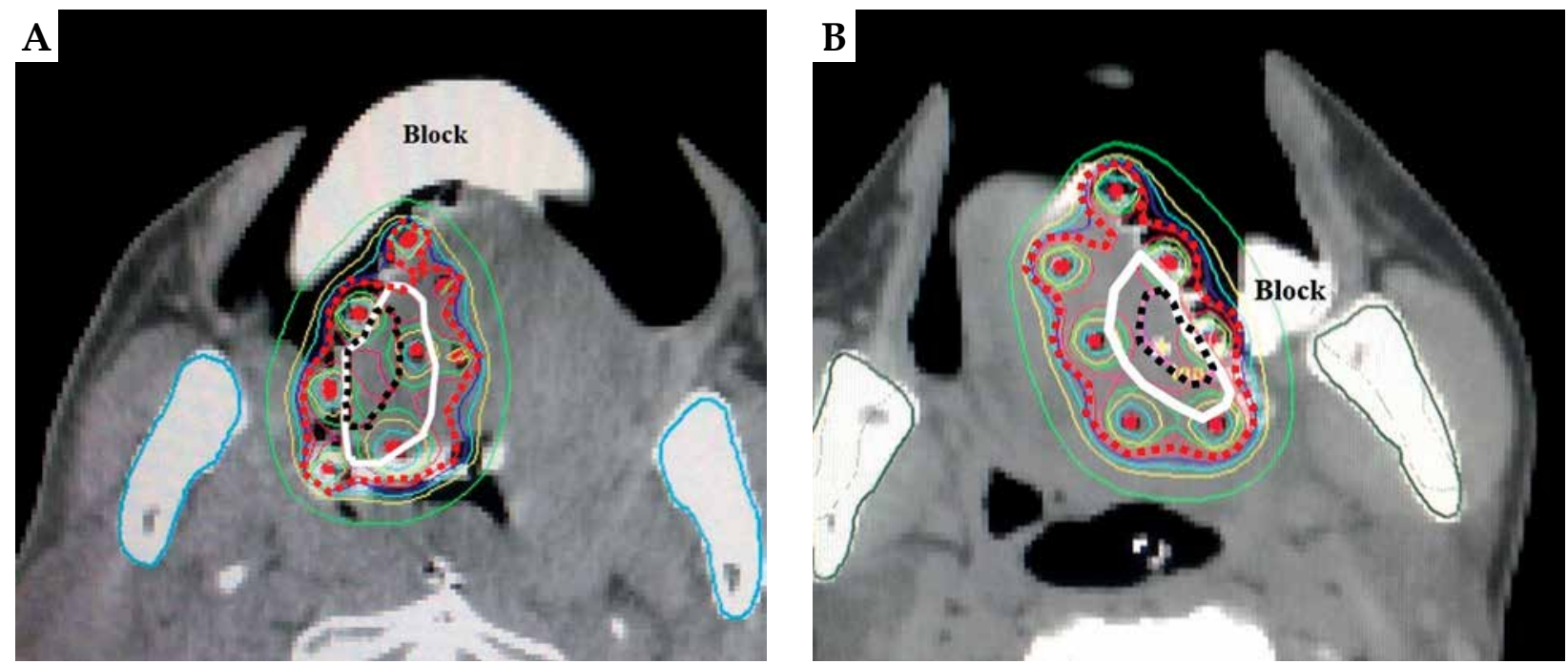

Fig. 3. A) An isodose shape for case 1. The gloss tumor volume (black dotted line) and clinical target volume (white line) were almost covered by the $100 \%$ prescribed isodose line (red dotted line). The silicone device was clearly visualized just laterally of the tongue. The silicone block (Block) was also visualized without any artifact. B) An isodose shape for case 2. The gloss tumor volume (black dotted line) and clinical target volume (white line) could be covered by the $100 \%$ prescribed isodose line (red dotted line). The silicone device was clearly visualized just laterally of the tongue. The silicone block (Block) was also visualized without any artifact 
day. The patients were not treated on Saturday and Sunday. This generates a biological effective dose $(\alpha / \beta=10)$ of $86.4 \mathrm{~Gy}$, and equals an equivalent total dose in 2-Gy fractions $\left(\mathrm{EQD}_{2}\right)$ of $72 \mathrm{~Gy}$. All treatments are performed using an iridium-192 ( ${ }^{192}$ Ir) HDR-afterloading system (microSelectron-HDR, Elekta AB, Stockholm, Sweden) with an apparent initial source activity of approximately $370 \mathrm{GBq}$. During the treatment period the implant devices were always put along the lateral border of the tongue.

\section{Clinical cases}

Between June and August 2012, two mobile tongue cancer patients were treated by HDR-ISBT using the new silicone device at National Hospital Organization Osaka National Hospital.

\section{Case 1}

An 84-year-old male patient with heavy smoking history presented in June 2012 at our department with squamous cell carcinoma on the right lateral border of the tongue. Tumor stage was CT2N0M0 according to the 2007 UICC classification system. After rejecting glossectomy as well as definitive external-beam radiotherapy (EBRT), he was offered HDR-ISBT as monotherapy (Figure 3A). Treatment consisted of 54.0 Gy delivered in nine fractional HDR doses of 6.0 Gy over 7 days. The volumetrically calculated GTV and CTV were $5.3 \mathrm{cc}$ and $17 \mathrm{cc}$, respectively. The generated $D_{90}(G T V)$ was 6.6 Gy and $D_{100}$ (GTV) 6.0 Gy. The respective $\mathrm{D}_{90}$ (CTV) was $6.3 \mathrm{~Gy}$ and $\mathrm{D}_{100}(\mathrm{CTV}) 5.0 \mathrm{~Gy}$ with $\mathrm{D}_{2 \mathrm{cc}}$ (mandible) at 3.4 Gy.

At 60 months after ISBT the patient remains free from disease without local or systemic progression. However, at 32 months after treatment he presented osteoradionecrosis of the mandible which requires pain management with oxycodone hydrochloride but no intervention or hospitalization. Against medical advice, he did not quit smoking despite experiencing symptomatic osteonecrosis [18].

\section{Case 2}

A 38-year-old female patient presented in August 2012 at our department with squamous cell carcinoma on the left lateral border of the tongue. Tumor stage was cT2N0M0 according to the 2007 UICC classification system. After rejecting glossectomy, she was offered HDRISBT as monotherapy (Figure 3B). Treatment consisted of 54.0 Gy delivered in nine fractional HDR doses of 6.0 Gy over 7 days. The volumetrically calculated GTV and CTV were $1.3 \mathrm{cc}$ and $6.1 \mathrm{cc}$, respectively. The generated $\mathrm{D}_{90}$ (GTV) was 6.6 Gy and $D_{100}$ (GTV) 6.3 Gy. The respective $\mathrm{D}_{90}(\mathrm{CTV})$ was $6.6 \mathrm{~Gy}$ and $\mathrm{D}_{100}(\mathrm{CTV}) 6.1 \mathrm{~Gy}$ with $\mathrm{D}_{2 \mathrm{cc}}$ (mandible) at $2.6 \mathrm{~Gy}$.

At 56 months after ISBT the patient remains free from local disease recurrence. However, 3 months after treatment she was diagnosed with cervical lymph node metastasis and received radical neck dissection with postoperative EBRT up to $60 \mathrm{~Gy}$ in 30 fractions. At present, she remains free from local or further systemic progression. No mandible complication was observed.

\section{Discussion}

Image-based HDR-ISBT has proven to be an effective modality in the treatment of tongue cancer by escalating the biologically effective dose to the treatment target whilst ameliorating conformity [1,2,3,4]. GEC-ESTRO ACROP recommendations [19] reported that brachytherapy alone has been nowadays replaced by surgery in the treatment of most T1 and T2 tumors due to advances in surgical and anesthetic procedures that have been proven safe and effective even in frail patients; however, no randomized trial has ever compared surgery versus brachytherapy in primary tumors. In addition, surgery provides a complete pathological documentation of the extent of disease that allows an individualized adjuvant treatment plan for subsequent radiotherapy. However, brachytherapy alone remains an acceptable mode of treatment in intact $\mathrm{T} 1$ and small $\mathrm{T} 2$ tumors with low risk of lymph node involvement that meets at least one of the 5 following criteria. Patient decision is one of the criteria, and our cases were applicable because they rejected glossectomy as well as definitive EBRT. 3D treatment planning in HDR enables anatomy-oriented dose optimization while the versatility of intratarget dose modulation inherent to ISBT can be controlled and directed to deliver higher doses to gross disease or to selectively reduce the dose to OARs [20]. However, the rapid dose fall-off in HDR can also become a cause of underdosage to the treatment target if tongue edema occurs. Schultze et al. [10] investigated the incidence and extent of tongue edema in 51 patients with predominantly base of tongue cancer, reporting an applicator-induced edematous deviation of tongue width of median $6.0 \mathrm{~mm}$ as measured by MRI. In our own experience, we also encountered tongue edema after interstitial tongue implantation which resulted in treatment target underdosage and eventually local disease recurrence.

In our previous study, the coverage of the lateral border of the tongue was $100 \%$ of the PD at first but it was decreased to $70 \%$ PD because part of the mucosal surface protruded between two flexible applicator tubes by tongue edema if we did not use the new implant device [13]. The $\mathrm{D}_{90}(\mathrm{CTV})$ values were $6.3 \mathrm{~Gy}(105 \% \mathrm{PD})$ and 6.6 Gy $(110 \%$ PD) in this study. In our previous study, the mean $\mathrm{D}_{90}$ (CTV) was $112.4 \pm 6.1 \%$ of the PD for the image-based plan [15]. And so, the DVH data of this study were reasonable if no displacement occurred.

In order to avoid such alterations in dosimetry, especially during fractionated treatments, we developed the described silicone device which is characterized by two significant merits. Firstly, the device can limit the anatomic extension of treatment-associated tongue edema within its own borders. It is clearly identifiable in CT imaging, therefore supporting target delineation and reproducible treatment planning (Figure 3A,B). Ideally it may be better to take CT images for accurate evaluation of edema and make a new plan at each fractionated treatment, but spine position is hard and tiring for patients because of difficulty swallowing due to irritation of the applicator tubes. Therefore we took CT images and made a plan only once. Edema can change the shape and vol- 
ume of the tongue in every direction. Our new devices prevent edema in lateral, anterior and dorsal sides. For the medial and posterior aspect of the tongue we applied flexible applicator tubes to maintain a sufficient safety margin from the GTV. Concerning the ventral side we took into account edema when we decided on dwell positions of the treatment source. Therefore we consider that edema-induced changes of tongue volume for all directions were reflected in our treatment. However, we will verify them one by one. Secondly, the device can serve as a template allowing even inexperienced brachytherapist to perform tongue implants with greater confidence and dosimetrically advantageous.

Notwithstanding, with regard to clinical outcomes the patient in case 1 developed symptomatic osteoradionecrosis. Even though $\mathrm{D}_{2 \mathrm{cc}}$ (mandible) was only 3.4 Gy per fraction and $30.6 \mathrm{~Gy}$ in terms of total physical dose, our device might have contributed to avoiding local disease recurrence but did not prevent OAR toxicity. It remains doubtful, however, whether a dose-effect relationship exists for such a relatively low dose or the necrosis was associated with continuation of smoking. Zevallos et al. [18] investigated 86 patients receiving EBRT for laryngopharyngeal cancer and reported that continuation of smoking was strongly associated with the development of osteoradionecrosis (relative risk [RR]: 1.32; 95\% confidence interval [CI]: 1.09-1.6, $p=0.03$ ).

We consider the introduced silicon device a meaningful tool which has the potential to keep an interstitial tongue implant three-dimensionally stable and to contribute to OARs sparing due to reproducible dosimetry. Its implementation and general rationale are obvious to the radiation oncologist. However, some aspects need to be emphasized concerning its applicability. First, the silicone device can only be positioned at the floor of the mouth without the option to use it for base of tongue lesions. In addition, it must be individually assembled for various clinical settings. This flexibility certainly extends its safe applicability but might also be an expenditure of time, albeit without relevance when considering its potential merits.

The limitation of this initial experience study is that the number of patients is only two, and it is difficult to draw firm conclusions. The other limitation of this study is the lack of evaluation of edema during treatment. Further studies with a larger number of patients using retrospective comparison of matched cohorts and edema evaluation by $\mathrm{CT}$ images at each ISBT are required.

\section{Conclusions}

The described silicone device has the potential to prevent underdosage to the CTV potentially related to treatment-associated tongue edema. It has been proven to be safe and easy to implement.

\section{Acknowledgements}

This work was supported by JSPS KAKENHI (Grantin-Aid for Scientific Research (C)) Grant Numbers JP17K10496, JP18K07639, JP17K10488, JP16K11534 and
JP15K15461. This work was also supported in part by Japan Agency for Medical Research and Development (JP18ck0106305h002, JP19ck0106305h003) and OMC Internal Research Grant.

We would like to thank Mineo Yoshida, MD, Shunsuke Miyake, RTT, Mari Mikami Ueda, RTT, Toshiro Kajihara, RTT, Katsuhito Kubo, RTT, the other staff of the departments of Radiology, Oral Surgery, Anesthesiology and nursing staff for helping us in many ways during the completion of this study.

\section{Disclosure}

The authors report no conflict of interest.

\section{References}

1. Inoue $T$, Teshima $T$, Murayama $S$ et al. Phase III trial of high and low dose rate interstitial radiotherapy for early oral tongue cancer. Int J Radiat Oncol Biol Phys 1996; 36: 1201-1204.

2. Inoue T, Teshima T, Yoshida K et al. Phase III trial of high- vs. low- dose-rate interstitial radiotherapy for early oral tongue cancer. Int J Radiat Oncol Biol Phys 2001; 51: 171-175.

3. Teshima $\mathrm{T}$, Inoue $\mathrm{T}$, Ikeda $\mathrm{H}$ et al. Phase I/II study of highdose rate interstitial radiotherapy for head and neck cancer. Strahlenther Onkol 1992; 168: 617-621.

4. Takácsi-Nagy Z, Polgár C, Oberna F et al. Interstitial highdose-rate brachytherapy in the treatment of base of tongue carcinoma. Strahlenther Onkol 2004; 180: 768-775.

5. Martinez AA, Pataki I, Edmundson G et al. Phase II prospective study of the use of conformal high-dose-rate brachytherapy as monotherapy for the treatment of favorable stage prostate cancer: a feasibility report. Int J Radiat Oncol Biol Phys 2001; 49: 61-69.

6. Damore SJ, Syed AM, Puthawala AA et al. Needle displacement during HDR brachytherapy in the treatment of prostate cancer. Int J Radiat Oncol Biol Phys 2000; 46: 1205-1211.

7. Cury FL, Duclos M, Aprikian A et al. Prostate gland edema after single-fraction high-dose rate brachytherapy before external beam radiation therapy. Brachytherapy 2010; 9: 208-212.

8. Kiffer JD, Schumer WA, Mantle CA et al. Impact of oedema on implant geometry and dosimetry for temporary high dose rate brachytherapy of the prostate. Australas Radiol 2003; 47: 172-176.

9. Yoshida K, Yamazaki H, Kotsuma T et al. Treatment results of image-guided high-dose-rate interstitial brachytherapy for pelvic recurrence of uterine cancer. Brachytherapy 2015; 14: 440-448.

10. Schultze J, Schneider R, Kimmig B et al. Interstitial brachytherapy in the head and neck region: when has imaging for treatment planning to be performed? Rontgenpraxis 2002; 54: 232-237.

11. Nag S, Cano ER, Demanes DJ et al. The American Brachytherapy Society recommendation for high-dose-rate brachytherapy for head-and-neck carcinoma. Int J Radiat Oncol Biol Phys 2001; 50: 1190-1198.

12. Mazeron JJ, Ardiet JM, Haie-Meder C et al. GEC-ESTRO recommendations for brachytherapy for head and neck squamous cell carcinomas. Radiother Oncol 2009; 91: 150-156.

13. Yoshida K, Yamazaki H, Kotsuma T et al. Edema worsens target coverage in high-dose-rate interstitial brachytherapy of mobile tongue cancer: a report of two cases. J Contemp Brachytherapy 2017; 9: 66-70.

14. Akiyama H, Yoshida K, Yamazaki H et al. High-dose-rate interstitial brachytherapy for mobile tongue cancer: preliminary results of a dose reduction trial. J Contemp Brachytherapy 2014; 6: 10-14. 
15. Yoshida K, Takenaka $\mathrm{T}$, Akiyama $\mathrm{H}$ et al. Three-dimensional image-based high-dose-rate interstitial brachytherapy for mobile tongue cancer. J Radiat Res 2014; 55: 154-161.

16. Yoshida K, Nose T, Koizumi M et al. The usefulness of metal markers for CTV-based dose prescription in high-dose-rate interstitial brachytherapy. J Jpn Soc Ther Radiol Oncol 2002; 13: 253-260.

17. Murakami S, Verdonschot RG, Kakimoto N et al. Preventing complications from high-dose rate brachytherapy when treating mobile tongue cancer via the application of a modular lead-lined spacer. PLoS One 2016; 11: e0154226.

18. Zevallos JP, Mallen MJ, Lam CY et al. Complications of radiotherapy in laryngopharyngeal cancer: effects of a prospective smoking cessation program. Cancer 2009; 115: 4636-4644.

19. Kovács G, Martinez-Monge R, Budrukkar A et al. GECESTRO Head \& Neck Working Group. GEC-ESTRO ACROP recommendations for head \& neck brachytherapy in squamous cell carcinomas: 1st update - Improvement by cross sectional imaging based treatment planning and stepping source technology. Radiother Oncol 2017; 122: 248-254.

20. Akiyama H, Major T, Polgár C et al. Dose-volume analysis of target volume and critical structures in computed tomography image-based multicatheter high-dose-rate interstitial brachytherapy for head and neck cancer. J Contemp Brachytherapy 2017; 9: 553-560. 